\title{
THE INITIAL MAGNETIC SUSCEPTIBILITY OF DENSE AGGREGATED DIPOLAR FLUIDS
}

\author{
SÁNDOR NAGY*1 \\ ${ }^{1}$ Institute of Mechatronics Engineering and Research, University of Pannonia, Gasparich M. u. 18/A, \\ Zalaegerszeg, $\mathrm{H}-8900$, HUNGARY
}

\begin{abstract}
To correlate the dipole moment and density dependence of the initial magnetic susceptibility on the basis of the former related theories and the probability analysis of chain formation, physically based analytical correlation equation was derived. After the local magnetic field strength and the chaining probability between two particle have been determined the chain and particle distributions came from the geometric distribution. The initial magnetic susceptibility was resulted from the summation of Langevin initial susceptibility of $k$-length chains. Two particles were considered in a chain if the interaction energy between them was below a certain limit. By varying slightly this energy limit around $70-75 \%$ good agreement has been obtained between the simulation and theoretical data. Monte Carlo simulations were used to calculate the initial magnetic susceptibility of dipolar hard sphere system at different dipole moments and densities.
\end{abstract}

Keywords: dipolar fluids, initial susceptibility, Monte Carlo simulation

\section{Introduction}

The investigation of dipolar fluids has been induced by the evolution of magnetorheology and electrorheology over the last two decades. The viscosity of electrorheological (ER) fluids increases dramatically due to an external electric field. ER fluids can be obtained by dispersing solid particles with dielectric permittivity $\epsilon_{\mathrm{p}}$ in a fluid with dielectric permittivity $\epsilon_{\mathrm{f}}$, where $\epsilon_{\mathrm{p}}>\epsilon_{\mathrm{f}}$. The dispersed particles are of between $0.1 \mathrm{~mm}$ and $100 \mathrm{~mm}$ in diameter. The polarized particles are organized into pairs and chains. The magnetic analogy of the phenomenon described above is the magnetorheological (MR) effect. If the magnetic permeabilities of the liquid and dispersed particles differ, then in an external magnetic field the particles are also arranged in chains. The dispersing medium can be water, oil, an organic solvent, etc. while the dispersed particles can be some kind of iron oxide or ferrit. In this paper, the magnetic terminology and centimetregram-second (CGS) system of units are used. In the figures, the reduced quantities are applied.

Electro- and magnetorheological fluids typically exhibit a reduced density of up to $\rho^{*}=0.4$ (where $\rho^{*}=$ $\rho \sigma^{3} ; \rho$ and $\sigma$ are the concentration and diameter of the suspended particles, respectively). The magnetic properties, e.g. magnetization curve and initial magnetic susceptibility, are well described by the various theories within this range of reduced density. The magnetization $\mathbf{M}$ can be obtained by summation of the dipole moments in the

\footnotetext{
*Correspondence: sata123.sandor@gmail.com
}

unit volume:

$$
\mathbf{M}=\frac{1}{V} \sum_{i} \mathbf{m}_{i} .
$$

In the absence of any external magnetic field the fluid is isotropic and according to Eq. 1 the magnetization is zero. When any external magnetic field is present, the field-oriented components of dipole moments should be summarized as

$$
\mathbf{M}=\rho m\langle\cos \Theta\rangle \frac{\mathbf{H}_{0}}{H_{0}},
$$

where $H_{0}=\left|\mathbf{H}_{0}\right|$ and $\langle\cos \Theta\rangle$ is the ensemble average of the cosine of the angle between $\mathbf{m}_{i}$ and $\mathbf{H}_{0}$, and $m=\left|\mathbf{m}_{i}\right|$. Since the directions of $\mathbf{H}_{0}$ and $\mathbf{M}$ are identical, vector notation can be omitted. The initial magnetic susceptibility is equal to the initial gradient of the magnetization curve

$$
\chi_{0}=\left.\frac{\partial M}{\partial H_{0}}\right|_{H_{0}=0} .
$$

In practice, ER and MR fluids can be used for the transmission of torque or force, in vibration dampers and braking systems, etc. The magnetic properties generally are calculated from Monte Carlo simulations because it is not necessary to know the velocity and acceleration of the particles nor the forces between them.

The expressions of the related models are listed in Table 1 . (One line belongs to one theory and the first line is the head of the table, e.g. Table 1: 2.4 refers to the 4 th cell in the 2 nd line within the 1 st table.) Three different 
Table 1: The expressions of the effective magnetic field, the magnetization and the initial magnetic susceptibility of the related theories.

\begin{tabular}{llrl}
\hline Model & $\begin{array}{l}H_{\mathrm{e}}-\text { Effective } \\
\text { magnetic field }\end{array}$ & $\begin{array}{l}M- \\
\text { Magnetization }\end{array}$ & $\begin{array}{l}\chi_{0} \text { - Initial } \\
\text { magnetic susceptibility }\end{array}$ \\
\hline $\begin{array}{l}\text { 1 "Langevin" } \\
\text { [1] }\end{array}$ & $H_{0}$ & $\rho m L\left(\frac{m H_{0}}{k_{\mathrm{B}} T}\right)$ & $\frac{\rho m^{2}}{3 k_{\mathrm{B}} T}=\chi_{\mathrm{L}}$ \\
$\begin{array}{l}\text { 2 "Weiss" } \\
\text { [2] }\end{array}$ & $H_{0}+\frac{4 \pi}{3} M\left(H_{\mathrm{e}}\right)$ & $\rho m L\left(\frac{m H_{\mathrm{e}}}{k_{\mathrm{B}} T}\right)$ & $\frac{\chi_{\mathrm{L}}}{1-\frac{4 \pi}{3} \chi_{\mathrm{L}}}$ \\
$\begin{array}{l}\text { 3 "Pshenichnikov" } \\
\text { [3] }\end{array}$ & $H_{0}+\frac{4 \pi}{3} M\left(H_{0}\right)$ & $\rho m L\left(\frac{m H_{\mathrm{e}}}{k_{\mathrm{B}} T}\right)$ & $\chi_{\mathrm{L}}\left(1+\frac{4 \pi}{3} \chi_{\mathrm{L}}\right)$ \\
$\begin{array}{l}\text { 4 "Ivanov" } \\
\text { [4] }\end{array}$ & $H_{0}+\frac{4 \pi}{3} M\left(H_{0}\right)\left(1+\frac{4 \pi}{48} \frac{\partial M}{\partial H_{0}}\right)$ & $\rho m L\left(\frac{m H_{\mathrm{e}}}{k_{\mathrm{B}} T}\right)$ & $\chi_{\mathrm{L}}\left(1+\frac{4 \pi}{3} \chi_{\mathrm{L}}+\frac{(4 \pi)^{2}}{144} \chi_{L}^{2}\right)$ \\
$\begin{array}{l}\text { 5 "Tani" and } \\
\text { "Szalai" [5, 6] }\end{array}$ & $M\left(H_{0}\right)=\rho m L+\frac{4 \pi}{3} \rho^{2} \beta m^{3} L L^{\prime}+\frac{1}{10} \rho^{2} \beta^{2} m^{5} \zeta^{\prime} I_{d d \Delta}$ & $\chi_{\mathrm{L}}\left(1+\frac{4 \pi}{3} \chi_{\mathrm{L}}+\frac{(4 \pi)^{2}}{144} \chi_{L}^{2} f(\rho)\right)$ \\
\hline
\end{tabular}

magnetic fields will be used. The applied external magnetic field is denoted by $H_{0}$ and the sum of the external and generated magnetic fields by $H_{\mathrm{e}} . H_{\mathrm{e}}$ is always parallel to $H_{0}$. The third one is the local magnetic field $H_{1}$ which is of chain-parallel orientation and its formation is due to dipole-dipole interactions between the particles.

The well-known Langevin function is applied from the initial theory [1] in the magnetization formula (Table 1: 1.3), where $L(\alpha)=\operatorname{coth} \alpha-1 / \alpha$. The magnetic dipole moment of the particles is denoted by $m$ and the applied external magnetic field by $H_{0}$, while the Boltzmann constant is represented by $k_{\mathrm{B}}$. The expression of magnetic susceptibility can be written as in Table 1: 1.4. This is known as Langevin susceptibility which is indicated by $\chi_{\mathrm{L}}$ as well. In this approach the effective magnetic field $H_{\mathrm{e}}$ exerted on the given particle is equal to the external magnetic field (Table 1: 1.2).

According to the more accurate model by Weiss [2] the effective magnetic field is equal to the sum of the external magnetic field and the magnetic field induced by the magnetization (Table 1:2.2). The formula of the magnetization (Table 1: 2.3) is similar to the previous one but $H_{0}$ is substituted by $H_{\mathrm{e}}$. Due to the iterative nature of the magnetization expression the initial magnetic susceptibility (Table 1: 2.4) exhibits divergence at $\chi_{\mathrm{L}}=3 / 4 \pi$, therefore, overestimates the real values. Above this initial magnetic susceptibility limit, when $\chi_{\mathrm{L}} \geq 3 / 4 \pi$, the zerofield magnetization is not equal to zero: $M\left(H_{0}\right) \nrightarrow \rightarrow+0$, if $H_{0} \rightarrow+0$. Moreover, in weak external magnetic fields, one $H_{0}$ value belongs to three equilibrium magnetization values.

The effective magnetic field has been substituted for the external magnetic field in the expression of the effective magnetic field (Table 1: 3.2) in the theory by Pshenichnikov et al. [3]. The magnetization formula (Table 1: 3.3 ) is the same as in Weiss' theory. The initial magnetic susceptibility (Table 1: 3.4) is in good agreement with the simulations but underestimates them at higher densities or higher dipole moments.

That is why it seems to be a good method to extend the expression of the effective magnetic field (Table 1: 4.2) by Ivanov et al. [4]. The magnetization formula is once again identical (Table 1: 4.3) but a new term is introduced in the initial magnetic susceptibility (Table 1: 4.4). Although at higher densities it yields higher values than in Pshenichnikov's model, it underestimates the simulation data as well. The factor of the third term is $(4 \pi)^{2} / 144=1.0966$ and perhaps it could be higher, but in this case at low densities the initial magnetic susceptibility overestimates the simulations.

The perturbation theory by Tani et al. [5] is worth mentioning because a density-dependent correction was used to complete the third term of the susceptibility (Table 1: 5.4), where $f(\rho)=9 I_{\mathrm{dd} \Delta} / \pi^{2}-16$, and

$$
I_{\mathrm{dd} \Delta}=\frac{17 \pi^{2}}{9}\left[\frac{1-0.93952 \rho^{*}+0.36714\left(\rho^{*}\right)^{2}}{1-0.92398 \rho^{*}+0.23323\left(\rho^{*}\right)^{2}}\right]
$$

The formula of the magnetization curve for this perturbation theory was calculated by Szalai et al. The expressions that are not mentioned in Table 1: 5.2 can be found in Ref. [6]. The values of the susceptibility more closely resemble the simulation data but still underestimate those.

It is worth mentioning the study by Huke and Lücke [7] who introduced the so-called "dipolar coupling constant" into the second term of the initial magnetic susceptibility, but the third term was ignored in expressions in Table 1: 4.4 and 5.4. Thereby their theory at higher densities underestimates and at lower densities overestimates the simulation data. Furthermore, the theory of mean-spherical approximation (MSA) [8-10] should also 
be mentioned which provides a formula for initial magnetic susceptibility and magnetization as well, but the validity of these are within the range of up to $m^{*}<\sqrt{1.5}$.

With regard to the distribution of chain aggregates in ferrofluids $[11,12]$, it has been found that in the absence of any external magnetic field, the chain size distribution is proportional to $p^{k} \exp (-\epsilon)$, where the chain length is denoted by $k$ and the dimensionless energy parameter $\epsilon$ is a function of the maximum dipole interaction energy but independent from the density, therefore, it is a constant here and the probability of bond formation between two adjacent particles in a chain is $p$. Subsequently, the chain size distribution decreases according to an exponential function because $p^{k}=\exp \left(-k / k_{0}\right)$, where $k_{0}=-1 / \ln p$. Based on some publications [13,14], in the case of high dipole moments this exponential expression turns into a power law: $g(k) \propto k^{-\iota}$, with exponent $\iota \approx 2.0-2.5$.

Our investigation was performed in a dipolar hardsphere (DHS) monodisperse system with a permanent magnetic dipole moment and of fixed density. It is supposed that the chains are perfectly straight and parallel to the local magnetic field. Furthermore, the average distance between two neighbouring particles in a chain is the same as the distance between two neighbouring parallel chains. The particles interact with each other only by the evolved mean magnetic field and the applied external magnetic field is superimposed on this, thus, the chains influence each other only by this mean magnetic field.

\section{Theory}

\subsection{The appearance of probability analysis in the initial magnetic susceptibility}

The distribution of chains was calculated with the aid of probability analysis in a zero applied magnetic field. As was mentioned in the "Introduction", Weiss' theory states that the effective (now "local") magnetic field converges to zero when $\chi_{\mathrm{L}}<3 / 4 \pi$ and non-zero values when $\chi_{\mathrm{L}} \geq 3 / 4 \pi$. The central and surrounding particles are under the influence of this local magnetic field. The chain is oriented in the same direction as the local magnetic field. Let us denote the probability of chain formation between two particles whose direction relative to each other is parallel to the local magnetic field by $p$.

Now using this approach the exact distribution of chain length can be calculated because the probability that the chain length ought to be equal to $k$ follows the geometric distribution with parameter $q$ :

$$
g_{k}=q p^{k-1},
$$

where $q=1-p$ and the "chain distribution" is denoted by $g_{k}$. According to its definition the geometric distribution shows the probability that a $k$ th particle is connected to a chain of length $k-1$ thus forming a chain of length $k$. A geometric sequence is described in Eq. 4, where the common ratio is denoted by $p$ and the first term by $q$. The sum of the terms of a geometric sequence is $S_{\infty}=\frac{f t}{1-c r}$, thus, $\sum_{k=1}^{\infty} g_{k}=\frac{q}{1-p}=\frac{q}{q}=1$.

It is also important to calculate the so-called "particle distribution" that implies the number of those particles which are members of the chains of length $k$ :

$$
h_{k}=q^{2} k p^{k-1} .
$$

The detailed deduction of $h_{k}$ and the sum of $h_{k}$ terms are described in Appendix A.

The expected value of the geometric distribution with parameter $q$ is $1 / q$, thus, here the average chain length is $1 / q$.

The number of chains is equal to the number of particles divided by the average chain length: $\frac{n}{1 / q}=n q$, where the number of particles is denoted by $n$.

Until now only the local magnetic field which arises from the strength of interaction energies between neighbouring particles and induces spontaneous magnetization in a random direction has been discussed, thus, the total magnetization of the system of volume $V$ is equal to zero.

When an infinitesimal external magnetic field $\mathbf{H}_{0}$ is switched on, non-zero total magnetization is formed. Since $\mathbf{H}_{0}$ is parallel to $\mathbf{M}$, scalar notations are used in the following. As was observed from Pshenichnikov's model the effective magnetic field is the sum of the external $H_{0}$ and secondary $(4 \pi / 3) M\left(H_{0}\right)$ magnetic fields. The question arises why it is legitimate to use the expression of effective magnetic field from "Pshenichnikov" (Table 1: 3.2) instead of from "Weiss" (Table 1: 2.2). The answer is because $H_{0}$ modifies infinitesimally the orientation of the chains but does not align them with its own direction, thus, the average angle between the local and external or even the effective magnetic fields is not equal to zero.

When calculating the initial magnetic susceptibility, a chain of length $k$ is considered as a particle with a dipole moment $\mathrm{km}$, thus, in terms of magnetization the argument of the Langevin function is $\frac{k m H_{\mathrm{e}}}{k_{\mathrm{B}} T}$. The Langevin function is weighted by the distribution $h_{k}$, and finally the gradient of magnetization in an infinitesimal external magnetic field is calculated as

$$
\chi_{0}=\left.\frac{\partial}{\partial H_{0}}\right|_{H_{0}=0} \rho m \sum_{k=1}^{\infty} h_{k} L\left(\frac{k m H_{\mathrm{e}}}{k_{\mathrm{B}} T}\right) .
$$

An infinitesimally weak external magnetic field can be written as

$$
\chi_{0}=\frac{1+p}{1-p} \chi_{\mathrm{L}}\left(1+\frac{4 \pi}{3} \chi_{\mathrm{L}}\right)
$$

where the following infinite expression is used $(|p|<1)$ :

$$
\sum_{k=1}^{\infty} p^{k-1} k^{2}=\frac{1+p}{(1-p)^{3}} .
$$

The detailed derivation of the initial magnetic susceptibility (Eq. 7) is given in Appendix B. 


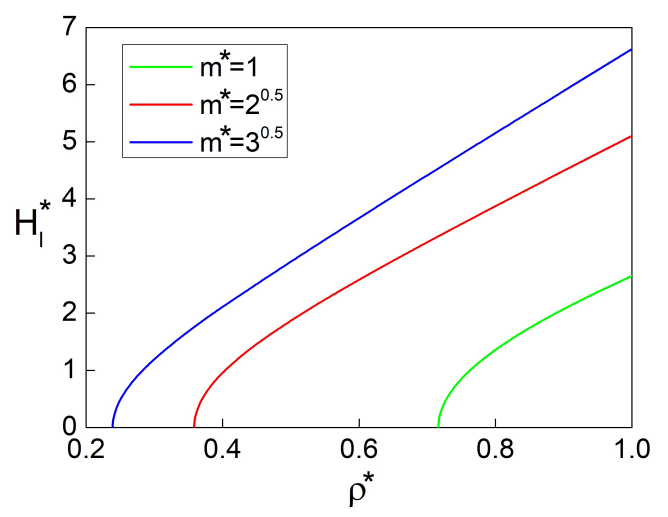

Figure 1: The rates of the local magnetic field as a function of the density from Eq. 9 at three different dipole moments in the absence of any external magnetic field.

\subsection{The numerical calculation of the probabil- ity of chain formation " $p$ "}

The main challenge of our approach is the determination of $p$. The particles form chains because of the local magnetic field even in the absence of any applied external magnetic field. According to Weiss' model when $\chi_{\mathrm{L}} \geq 3 / 4 \pi$ this local magnetic field predicts an infinite initial magnetic susceptibility. The problem with this model is that it assumes that the orientation of the local magnetic field is parallel with the external magnetic field. Nevertheless, Weiss' model is applicable to predict the extent of the local magnetic field by the expression (when $H_{0}=0$ ):

$$
H_{\mathrm{l}}=\frac{4 \pi}{3} \rho m L\left(\frac{m H_{1}}{k_{\mathrm{B}} T}\right) .
$$

$H_{1}^{*}$ as a function of the reduced density $\rho^{*}$ at three different dipole moments is presented in Fig. 1. The definitions of the reduced quantities are $H^{*}=H \sqrt{\sigma^{3} / k_{\mathrm{B}} T}$; $M^{*}=M \sqrt{\sigma^{3} / k_{\mathrm{B}} T} ; m^{*}=m / \sqrt{\sigma^{3} k_{\mathrm{B}} T}$.

All particles are considered to be influenced by this local magnetic field $H_{1}$, in the absence of any external magnetic field $H_{0}$ when calculating the initial magnetic susceptibility. As was mentioned before, the most accepted criterion for chaining is to determine an energy level and if the dipolar energy between two given particles is under this level, the particles are in a bound relationship. Generally [15-17], this energy level is 70-75 $\%$ of the minimum of the dipolar energy, i.e. $U_{\lim }^{*}=$ $-0.7 * 2\left(m^{*}\right)^{2}$.

Here the well-known dipolar energy is defined as the interaction between point dipoles:

$$
U_{i j}^{\mathrm{dd}}=-\frac{m^{2}}{r_{i j}^{3}}\left[3\left(\widehat{\mathbf{m}}_{i} \cdot \widehat{\mathbf{r}}_{i j}\right)\left(\widehat{\mathbf{m}}_{j} \cdot \widehat{\mathbf{r}}_{i j}\right)-\left(\widehat{\mathbf{m}}_{i} \cdot \widehat{\mathbf{m}}_{j}\right)\right],
$$

where the particles have dipole moments of strength $m$ as well as an orientation given by unit vectors $\widehat{\mathbf{m}}_{i}$ and

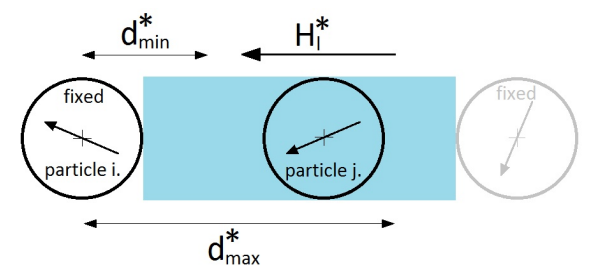

Figure 2: The feasible location of a particle between two fixed particles.

$\widehat{\mathbf{m}}_{j}$. Furthermore, the distance between the centers of the particles is denoted by $r_{i j}$ and $\widehat{\mathbf{r}}_{i j}=\mathbf{r}_{i j} / r_{i j}$.

As is shown in Fig. 2, according to our model particle $j$ can move along the direction of the chain between the two fixed adjacent particles, namely $i$ and the grey one, in the tube with a light blue background. Logically, the minimum distance between two particles in a hard sphere system is $\sigma$, on a reduced scale $d_{\min }^{*}=1$, while for the maximum distance $d_{\max }^{*}=2\left\langle r^{*}\right\rangle-1$, where the reduced average distance between two adjacent particles is denoted by $\left\langle r^{*}\right\rangle$.

Obviously the maximum distance between two neighbouring particles could be greater than $d_{\max }^{*}$ but at higher densities in particular the surrounding particles obstruct the movement of the central particle. Assuming that the distance distribution is isotropic, it is given by $\left\langle r^{*}\right\rangle=$ $\sqrt[3]{1 / \rho^{*}}$

Taken all round to calculate $p$ the probability of those states of particle pairs should be totalled when the dipolar interaction energy is less than or equal to the aforementioned energy limit $U_{\text {lim }}$ and the interval of integration in distance is $\left[d_{\min }^{*}, d_{\max }^{*}\right]$, that is

$$
p=\int_{U^{\mathrm{dd}} \leq U_{\lim }} P\left(\theta_{i}\right) d \theta_{i} P\left(\theta_{j}\right) d \theta_{j} P\left(\phi_{i}\right) d \phi_{i} P\left(\phi_{j}\right) d \phi_{j},
$$

where $0 \leq \theta<\pi$ and $0 \leq \phi<2 \pi$ are the usual spherical angles of the dipoles and the probabilities when magnetic field $H$ (here $H=H_{1}$ ) is applied in general are

$$
P(\theta) d \theta=\frac{\exp \left(\frac{m H}{k_{\mathrm{B}} T} \cos \theta\right) \sin \theta d \theta}{\int_{0}^{\pi} \exp \left(\frac{m H}{k_{\mathrm{B}} T} \cos \theta\right) \sin \theta d \theta}
$$

and $P(\phi) d \phi=d \phi / 2 \pi$.

The calculation of $p$ was performed by numerical integration. Particles $i$ and $j$ (Fig. 2) are under the influence of the local magnetic field independently from each other. For both particles, all possible values of $\theta, \phi$, and $r$ are swept and taken into account if the dipolar interaction energy between particles $i$ and $j$ is less than or equal to $U_{\text {lim. }}$. This is expressed by Eq. 11 .

For instance, when $\rho^{*}=0.8$ and $m^{*^{2}}=3.0$ then $H_{l}^{*}=5.154$ and $d_{\max }^{*}=1.154435$. The probability of chaining between particles $i$ and $j$ as a function of distance is shown in Fig. 3. The requested probability $p$ is 


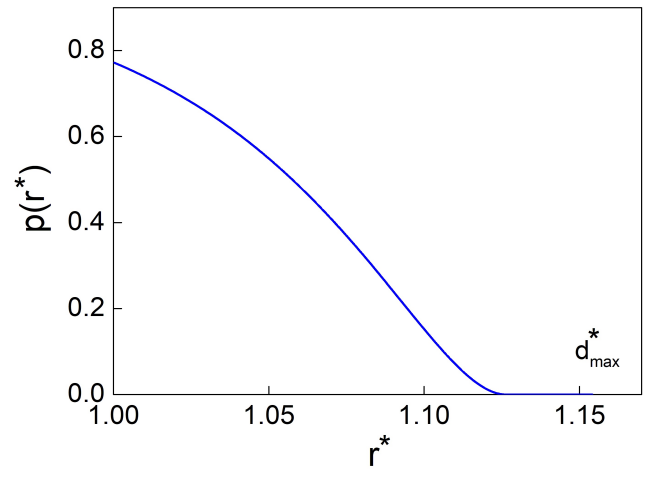

Figure 3: An example calculation of $p$. The parameters are $r h o^{*}=0.8,\left(m^{*}\right)^{2}=3.0, H_{l}^{*}=5.154$, $d_{\max }^{*}=1.154435$, and $U_{\lim }^{*}=0.7 U_{\min }^{*}$. The requested probability $p$ is the average of this curve in the interval $\left[d_{\min }^{*}, d_{\max }^{*}\right]$.

the average of this curve from 1 to $d_{\max }^{*}$, i.e. $p=0.3454$, if $U_{\lim }^{*}=0.7 U_{\min }^{*}$.

\section{Simulation results and discussion}

To determine the initial magnetic susceptibility, Monte Carlo simulations of DHS fluids were performed using a canonical NVT ensemble. Boltzmann sampling [18], periodic boundary conditions and the minimum-image convention were applied. In order to take into account the long-range character of the dipolar interaction, the reaction field method under boundary conditions of conduction was used. After 100,000 equilibration cycles, between 1 and 10 million production cycles were conducted involving $N=512$ particles. In the absence of an external magnetic field, the initial magnetic susceptibility was obtained from the following fluctuation formula:

$$
\chi_{0}=\frac{1}{3 k_{\mathrm{B}} T V}\left(\left\langle\mathbf{M}^{2}\right\rangle_{0}-\langle\mathbf{M}\rangle_{0}^{2}\right),
$$

where $\mathbf{M}=\sum_{i=1}^{N} \mathbf{m}_{i}$.

The exact results of the probability of chain formation from Eq. 11 applied to the local magnetic field, given by Eq. 9, are shown in Table 2. The data associated with the aforementioned dipole moments were rounded to three non-zero decimals. According to Fig. 1 at low densities the values of the local magnetic field are zero, nevertheless, the rates of the probability of chaining are not equal to zero. The value of the energy limit was fitted to the best agreement between the simulation data and our theory lines.

Our theoretical findings (green lines, Eq. 7 ) in terms of the initial magnetic susceptibility according to our Monte Carlo simulation data (blue dots) and the values of Ivanov's theory (grey lines, Table 1: 4.4) are compared in Figs. 4-6. The variability is not indicated where its magnitude is comparable to the size of the dot. The values of dipole moments in the order $m^{*}=1, m^{*}=\sqrt{2}$ and $m^{*}=\sqrt{3}$ are shown in Figs. 4-6.
Table 2: The probability of chaining at three different dipole moments. The applied energy limit at $m^{*}=1$ and $m^{*}=\sqrt{2}$ is $U_{\lim }=0.77 U_{\min }$ and $U_{\lim }=0.71 U_{\min }$ at $m^{*}=\sqrt{3}$.

\begin{tabular}{llll}
\hline \multicolumn{3}{c}{$p$} \\
\hline$\rho^{*}$ & $m^{*}=1$ & $m^{*}=\sqrt{2}$ & $m^{*}=\sqrt{3}$ \\
\hline 0.1 & 0.000260 & 0.000263 & 0.000580 \\
0.2 & 0.000426 & 0.000429 & 0.000943 \\
0.3 & 0.000615 & 0.000617 & 0.00794 \\
0.4 & 0.000852 & 0.00292 & 0.0250 \\
0.5 & 0.00117 & 0.0110 & 0.0521 \\
0.6 & 0.00164 & 0.0253 & 0.0953 \\
0.7 & 0.00241 & 0.0515 & 0.170 \\
0.8 & 0.0135 & 0.107 & 0.320 \\
0.85 & 0.0269 & 0.164 & 0.471 \\
0.9 & 0.0539 & 0.273 & 0.649 \\
0.95 & 0.101 & 0.416 & 0.775 \\
\hline
\end{tabular}

In the case of $m^{*}=1$ (Fig. 4), the energy limit is $U_{\lim }=0.77 U_{\min }$. A significant difference was only observed between Ivanov's theory and the simulation data beyond a reduced density of 0.8 and the simulation dots were tracked by our present theory. The maximum relative deviation from the simulation data is $\left|\chi_{0 \operatorname{sim}}-\chi_{0 \text { th }}\right| / \chi_{0 \operatorname{sim}}=4.175 \%$.

When $m^{*}=\sqrt{2}$, the appropriate energy criterion is $U_{\text {lim }}=0.77 U_{\min }$ as well. Up to a reduced density of 0.6 the former theory and simulation are in good agreement (Fig. 5), but in the present theory more than double the surplus is shown in the region of high density compared to Table 1: 4.4. The maximum relative deviation from the simulation data is $8.024 \%$.

Here it is quite conceivable that the simple series expansion of initial magnetic susceptibility as the summation of positive integer powers of Langevin susceptibility is unsatisfactory. By increasing the third coefficient in

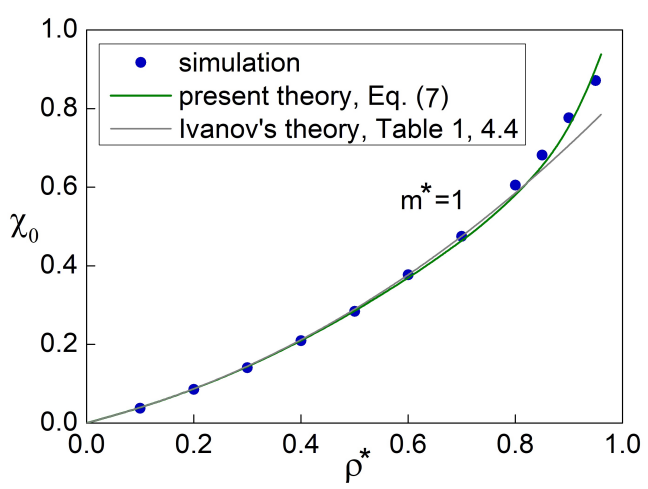

Figure 4: Initial magnetic susceptibility of DHS fluid as a function of reduced density with dipole moment $m^{*}=1$. Monte Carlo simulation results are denoted by symbols and the solid lines correspond to the present (Eq. 7) and Ivanov's (Table 1: 4.4) theories. 


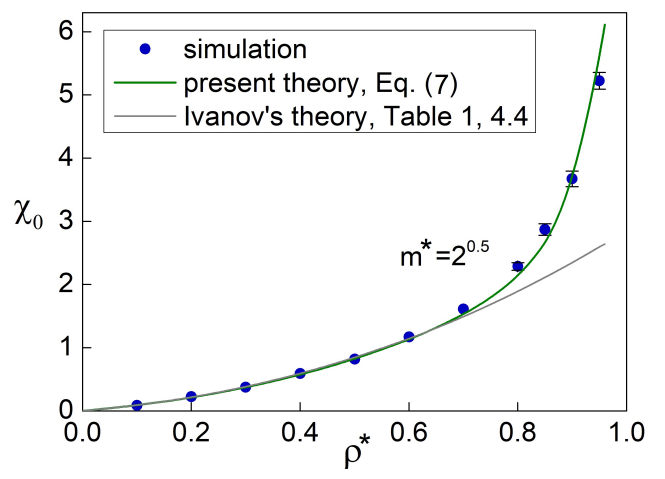

Figure 5: Initial magnetic susceptibility of DHS fluid as a function of reduced density with dipole moment $m^{*}=$ $\sqrt{2}$. Monte Carlo simulation results are denoted by symbols and the solid lines correspond to the present (Eq. 7) and Ivanov's (Table 1:4.4) theories.

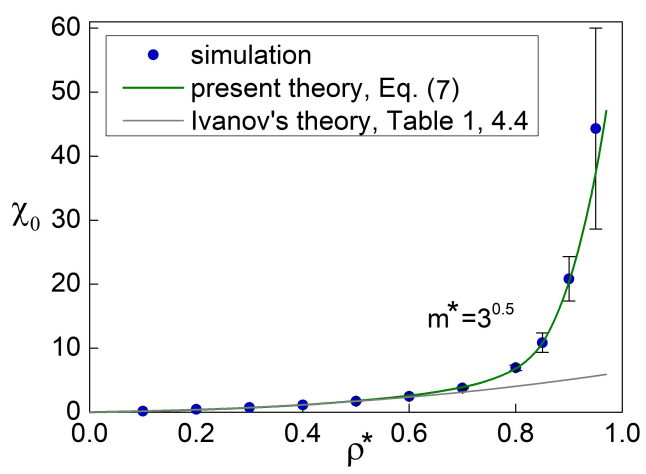

Figure 6: Initial magnetic susceptibility of DHS fluid as a function of reduced density with dipole moment $m^{*}=$ $\sqrt{3}$. Monte Carlo simulation results are denoted by symbols and the solid lines correspond to the present (Eq. 7) and Ivanov's (Table 1: 4.4) theories.

Table 1: 4.4, the initial magnetic susceptibility at lower densities is also increased.

When $m^{*}=\sqrt{3}$ the difference is even more spectacular between the theories (Fig. 6). At high densities the uncertainty of initial magnetic susceptibility is quite large with regard to the simulation data. The best fit curve belongs to an energy limit of $71 \%$, which is very close to the value from references [15-17] of $70 \%$. The maximum relative deviation from the simulation data is 15.850 $\%$.

When $m^{*}=1$ and $\rho^{*}=0.95$, with an energy limit of $70 \%$, the theoretical value of initial magnetic susceptibility is $\chi_{0}=1.042$, and if the energy limit is $75 \%$, $\chi_{0}=0.937$. Both values are higher than the corresponding simulation data, thus, the energy limit has to be raised to $77 \%$. The situation is similar when $m^{*}=\sqrt{2}$ and $\rho^{*}=0.95$, namely $\chi_{0}=8.701$ when the energy limit is $70 \%$ and $\chi_{0}=6.363$ when it is $75 \%$.

Probably at lower dipole moments and higher densities the two adjacent particles cannot be considered as a chain even though the interaction energy exceeds an energy limit of $70 \%$ or $75 \%$ for example because the average kinetic energy is closer to this interaction energy than is the case when $m^{*}=\sqrt{3}$. Therefore, the duration of chain formation is short to draw the particles together.

\section{Conclusion}

The initial magnetic susceptibility of dipolar hard sphere fluids was described by the help of the probability variable $p$ supplemented by Pshenichnikov's well-known theory. The validity of the present theory is up to $\rho^{*}=0.95$ and at least $m^{*}=\sqrt{3}$. In addition to the theoretical work, Monte Carlo simulations were run to confirm our investigation. At higher densities, especially with higher dipole moments, the former related theories significantly underestimate the simulation data but good results are also provided by the presented theory within this range. By considering the green curves and blue dots in Figs. 4-6, it is obvious that the simple quadratic or tertiary polynomial approach is outdated, therefore, the Taylor series expansion of $(1+p) /(1-p)$ contains powers even as high as infinity.

\section{Appendix}

\subsection{Appendix $A$}

The particle distribution can be obtained by dividing the number of particles in chains of length $k$ by the total number of particles:

$$
\begin{aligned}
& h_{k}=\frac{k g_{k}}{\sum k g_{k}}=\frac{k q p^{k-1}}{\sum k q p^{k-1}}=\frac{k p^{k-1}}{\sum k p^{k-1}}=\frac{k p^{k-1}}{1+2 p+3 p^{2}+\cdots}= \\
= & \frac{k p^{k-1}}{\left(1+p+p^{2}+\cdots\right)+\left(p+p^{2}+p^{3}+\cdots\right)+\left(p^{2}+p^{3}+p^{4}+\ldots\right)+\cdots}= \\
= & \frac{k p^{k-1}}{\left(1+p+p^{2}+\cdots\right)+\left(p+p^{2}+p^{3}+\cdots\right)+\left(p^{2}+p^{3}+p^{4}+\cdots\right)+\cdots}= \\
= & \frac{k p^{k-1}}{\frac{1}{1-p}+\frac{p}{1-p}+\frac{p^{2}}{1-p}+\cdots}=\frac{q k p^{k-1}}{1+p+p^{2}+\cdots}=q^{2} k p^{k-1} \\
& \frac{1}{1-p}
\end{aligned}
$$


The sum of $h_{k}$ terms must be equal to one:

$$
\begin{gathered}
\sum_{k=1}^{\infty} h_{k}=\sum_{k=1}^{\infty} q^{2} k p^{k-1}=q^{2}\left(1+2 p+3 p^{2}+\ldots\right)=q^{2}\left[\left(1+p+p^{2}+\cdots\right)+\left(p+p^{2}+p^{3} \cdots\right)+\cdots\right]= \\
=q^{2}\left[\frac{1}{1-p}+\frac{p}{1-p}+\frac{p^{2}}{1-p}+\cdots\right]=q\left[1+p+p^{2}+\ldots\right]=q \frac{1}{1-p}=1
\end{gathered}
$$

\subsection{Appendix $B$}

$$
\begin{gathered}
\chi_{0}=\left.\frac{\partial}{\partial H_{0}}\right|_{H_{0}=0} \rho m \sum_{k=1}^{\infty} h_{k} L\left(\frac{k m H_{e}}{k_{\mathrm{B}} T}\right)=\left.\frac{\partial}{\partial H_{0}}\right|_{H_{0}=0} \rho m \sum_{k=1}^{\infty} q^{2} k p^{k-1} L\left(\frac{k m\left(H_{0}+\frac{4 \pi}{3} \rho m L\left(\frac{m H_{0}}{k_{\mathrm{B}} T}\right)\right)}{k_{\mathrm{B}} T}\right)= \\
=\left.q^{2} \rho m \frac{\partial}{\partial H_{0}}\right|_{H_{0}=0} \sum_{k=1}^{\infty} k p^{k-1} L\left(\frac{k m H_{0}}{k_{\mathrm{B}} T}+\frac{\frac{4 \pi}{3} k \rho m^{2} L\left(\frac{m H_{0}}{k_{\mathrm{B}} T}\right)}{k_{\mathrm{B}} T}\right)= \\
=q^{2} \rho m \sum_{k=1}^{\infty} k p^{k-1} \frac{1}{3}\left(\frac{k m}{k_{\mathrm{B}} T}+\frac{\frac{4 \pi}{3} k \rho m^{2} \frac{1}{3} \frac{m}{k_{\mathrm{B}} T}}{k_{\mathrm{B}} T}\right)=q^{2} \sum_{k=1}^{\infty} k^{2} p^{k-1}\left(\frac{1}{3} \frac{\rho m^{2}}{k_{\mathrm{B}} T}+\frac{1}{9} \frac{4 \pi}{3} \frac{\rho^{2} m^{4}}{k_{\mathrm{B}}^{2} T^{2}}\right)= \\
q^{2}\left(\chi_{\mathrm{L}}+\frac{4 \pi}{3} \chi_{\mathrm{L}}^{2}\right) \sum_{k=1}^{\infty} k^{2} p^{k-1}=\frac{1+p}{1-p} \chi_{\mathrm{L}}\left(1+\frac{4 \pi}{3} \chi_{\mathrm{L}}\right) .
\end{gathered}
$$

\section{REFERENCES}

[1] Langevin, P.: Sur la théorie du magnétisme, Journal de Physique Théorique et Appliquée, 1905 4(1), 678-693 DOI: 10.1051/jphystap:019050040067800

[2] Weiss, P.: L'hypothése du champ moléculaire et la propriété ferromagnétique, Journal de Physique Théorique et Appliquée, 1907 6(1), 661-690 DOI: 10.1051/jphystap:019070060066100

[3] Pshenichnikov, A. F.; Mekhonoshin, V. V.: Equilibrium magnetization and microstructure of the system of superparamagnetic interacting particles: numerical simulation, Journal of Magnetism and Magnetic Materials, 2000 213(3), 357-369 DOI: 10.1016/S0304-8853(99)00829-X

[4] Ivanov, A. O.; Kuznetsova, O. B.: Magnetic properties of dense ferrofluids: An influence of interparticle correlations, Phys. Rev. E, 2001 64(4), 041405 DOI: 10.1103/PhysRevE.64.041405

[5] Tani, A.; Henderson, D.; Barker, J. A.; Hecht, C. E.: Application of perturbation theory to the calculation of the dielectric constant of a dipolar hard sphere fluid, Mol. Phys., 1983 48(4), 863-869 DOI: 10.1080/00268978300100621

[6] Szalai, I.; Nagy, S.; Dietrich, S.: Linear and nonlinear magnetic properties of ferrofluids, Phys. Rev. E, 2015 92(4), 042314 DOI: 10.1103/PhysRevE.92.042314
[7] Huke, B.; Lücke, M.: Magnetization of ferrofluids with dipolar interactions: A Born-Mayer expansion, Phys. Rev. E, 2000 62(5), 6875-6890 DOI: 10.1103/PhysRevE.62.6875

[8] Wertheim, M. S.: Exact solution of the mean spherical model for fluids of hard spheres with permanent electric dipole moments, J. Chem. Phys., 1971 55(9), 4291-4298 DOI: 10.1063/1.1676751

[9] Hansen, J.-P.; McDonald, I. R.: Theory of Simple Liquids (Elsevier, New York) 2005 ISBN: 9780123705358

[10] Szalai, I.; Dietrich, S.: Magnetization and susceptibility of ferrofluids, J. Phys.: Cond. Matt., 2008 20(20), 204122 DOI: 10.1088/0953-8984/20/20/204122

[11] Zubarev, A. Y.; Iskakova, L. Y.: Theory of physical properties of magnetic liquids with chain aggregates, J. Exp. Theor. Phys., 1995 80(5), 857-866

[12] Zubarev, A. Y.; Iskakova, L. Y.: Effect of chainlike aggregates on dynamical properties of magnetic liquids, Phys. Rev. E, 2000 61(5), 5415-5421 DOI: 10.1103/PhysRevE.61.5415

[13] Stauffer, D.; Aharony, A.: Introduction to percolation theory (CRC Press, London) 1994 ISBN: 9780748402533

[14] Del Gado, E.; Kob, W.: A microscopic model for colloidal gels with directional effective interactions: network induced glassy dynamics, Soft Matter, 2010 6(7), 1547-1558 DOI: 10.1039/B916813C 
[15] Weiss, J. J.; Levesque, D.: Chain formation in low density dipolar hard spheres: A Monte Carlo study, Phys. Rev. Lett., 1993 71(17), 2729-2732 DOI: 10.1103/PhysRevLett.71.2729

[16] Levesque, D.; Weis, J. J.: Orientational and structural order in strongly interacting dipolar hard spheres, Phys. Rev. E, 1994 49(6), 5131-5140 DOI:
10.1103/PhysRevE.49.5131

[17] Stevens, M. J.; Grest, G. S.: Structure of soft-sphere dipolar fluids, Phys. Rev. E, 1995 51(6), 5962-5975 DOI: 10.1103/PhysRevE.51.5962

[18] Allen, M. P.; Tildesley D. J.: Computer Simulation of Liquids (Clarendon Press, Oxford) 1987 ISBN: 978-0198556459 